\title{
Progreso Técnico y Poder de Mercado en la Industria Textil: Evidencia Empírica para Colombia, 1975- 2006
}

\author{
José B. Gallego-Alzate ${ }^{1}$ \\ Jaime A. Osorio-Usuga ${ }^{2}$ \\ Juan D. Lora-Restrepo ${ }^{3}$
}

\section{Resumen}

Este trabajo presenta los resultados de un estudio relacionado con la existencia de poder de mercado en la industria textil colombiana desde 1975 hasta 2006. Se utiliza como indicador de poder de mercado el índice de Lerner, obtenido a partir de la generalización del residuo de Solow. La estimación se realiza utilizando series de tiempo obtenidas de la Encuesta Anual Manufacturera y aplicando el método de los mínimos cuadrados ordinarios. La conclusión fundamental es la confirmación de la existencia de poder de mercado en la industria textil, al estimarse un índice de Lerner mayor que cero y un markup de Hall mayor que uno.

\section{Palabras clave}

Poder de mercado, costo marginal, índice de Lerner, residuo de Solow, industria textil.

1 Facultad de Ingenierías, Instituto Tecnológico Metropolitano, Medellín-

Colombia, josegallego@itm.edu.co

2 Dirección de Desarrollo Territorial, Departamento Nacional de Planeación, Bogotá-Colombia, jaimeusuga@gmail.com

3 Planeación Financiera, Sadinsa S.A., Bogotá-Colombia, lorarestrepo@gmail.com

Fecha de recepción: 21 de junio de 2011

Fecha de aceptación: 18 de octubre de 2011 
[140] Progreso Técnico y Poder de Mercado en la Industria Textil: Evidencia Empírica para Colombia, 1975-2006

\section{Abstract}

In this paper, the estimation of market power in the Colombian textile industry during 1975-2006 is presented. It is applied as indicator Lerner index based on Solow's residual, the information is of the manufacturing annual survey and method estimation is the square ordinary minimums. The key conclusion is confirmation of the existence of market power, estimated at a rate of greater than zero Lerner index and Hall markup of more than one.

\section{Keywords}

Market power, cost margin, Lerner index, Solow's residual, textile industry. 


\section{INTRODUCCIÓN}

El sector textil comprende la elaboración de hilos e hilazas y la elaboración de tejido plano y de punto a partir de fibras de origen animal, vegetal o mineral. Este renglón de actividad es de suma importancia para la economía colombiana dada su participación del $16,36 \%$ en el producto total de la industria manufacturera durante el año 2005 y sus procesos intensivos en mano de obra, los cuales generan el $22,74 \%$ del empleo dentro de la industria, de acuerdo con la Encuesta Anual Manufacturera (DANE, 2006), el cambio técnico incorporado, la participación sobre las exportaciones totales, entre otros factores dan cuenta de la importancia del proceso de producción textilera en la estructura productiva de Colombia. Por tal motivo, el estudio sobre su poder de mercado, su organización y nivel de concentración se hace relevante; más aún si consideramos que no se cuenta con investigaciones que analicen este fenómeno a la luz de cambios estructurales como la apertura económica de la década de 1990.

Este artículo presenta los resultados de una investigación relacionada con la existencia de poder de mercado en la industria textil colombiana durante 1975-2006. El estudio se realiza bajo el enfoque de la denominada Nueva Organización Industrial Empírica. En particular, el trabajo se apoya en una variación del modelo desarrollado por (Hall, 1988) fundamentado en el concepto de residuo de Solow (Solow, 1957). La variación fue desarrollada por (Linnosmaa et. al., 2004), y es el modelo estimado en esta investigación. Las series de tiempo para el análisis econométrico son obtenidas con base en la información sectorial recopilada por el DANE a través de la Encuesta Anual Manufacturera.

El objetivo es determinar la existencia de poder de mercado en el sector textil colombiano durante el período 1975-2006 a partir de la estimación del margen precio-costo marginal. Para el logro del objetivo, el presente trabajo consta de la presente introducción, luego se presenta la estructuración teórica del modelo a desarrollar, los resultados de la estimación, un análisis de las estimaciones obtenidas y finalmente las conclusiones. Como resultados adicionales, se calcula el residuo de Solow, el costo marginal de la 
industria, la elasticidad precio de la demanda y el crecimiento del progreso técnico.

\section{ESTRUCTURACIÓN TEÓRICA}

\subsection{El Residuo de Solow en Mercados Competitivos}

Para obtener el residuo de Solow en mercados competitivos se parte de los siguientes supuestos: la estructura de mercado es de competencia perfecta para los productos y los factores; el cambio técnico es neutral; y una tecnología en la que la función de producción exhibe rendimientos constantes a escala en sus factores. Para estimar el progreso técnico o residuo de Solow, se parte de una función de producción con progreso técnico y dos factores de producción, así:

$Q_{t}=A_{t} f\left(K_{t}, L_{t}\right)$

donde $Q$ es el volumen de producción (producto total); $f$ representa la función de producción; $K$ es la medida del insumo capital; $L$ es la cantidad de trabajo medido en unidades físicas, y la variable $A$ representa el progreso técnico. Además, el tiempo no aparece directamente en la función de producción, sino que lo hace a través de las variables $Q, A, L, K$, indicando que la producción varía en el tiempo sólo si lo hacen las variables que la determinan (Romer, 2001).

Derivando a la función de producción respecto al tiempo (Gallego, 2009)

$$
\frac{\partial Q_{t}}{\partial t}=\frac{\partial A_{t}}{\partial t} \frac{Q_{t}}{A_{t}}+A_{t}\left[\frac{\partial f\left(K_{t}, L_{t}\right)}{\partial K_{t}} \frac{\partial K_{t}}{\partial t}+\frac{\partial f\left(K_{t}, L_{t}\right)}{\partial L_{t}} \frac{\partial L_{t}}{\partial t}\right]
$$

Al incluir en (2) la notación para tasas de crecimiento y multiplicando por un factor uno (por $\frac{K_{t}}{K_{t}}$ y por $\frac{L_{t}}{L_{t}}$ ), se obtiene: 


$$
\frac{\dot{Q}}{Q_{t}}=\frac{\dot{A}}{A_{t}}+\frac{A_{t}}{Q_{t}} \frac{\partial f\left(K_{t} L_{t}\right) K_{t}}{\partial K_{t}} \frac{\dot{K}}{K_{t}}+\frac{A_{t}}{Q_{t}} \frac{\partial f\left(K_{t}, L_{t}\right) L_{t}}{\partial L_{t}} \frac{\dot{L}}{L_{t}}
$$

En competencia perfecta el precio es igual al costo marginal; además el trabajo y el capital son remunerados según sus respectivas productividades marginales. Siendo $c$ el costo marginal, $p$ el precio de mercado del producto, $w$ el salario nominal y $r$ la compensación al capital. En competencia perfecta, las remuneraciones reales a los factores de producción son el salario real (4) y la remuneración real al capital (5)

$$
\begin{aligned}
& \frac{w}{p}=A \frac{\partial f(K, L)}{\partial L} \\
& \frac{r}{p}=A \frac{\partial f(K, L)}{\partial K}
\end{aligned}
$$

Reemplazando lo anterior en (3), ésta se puede reescribir como:

$$
\frac{\dot{Q}}{Q}=\frac{\dot{A}}{A}+\frac{r K}{p Q} \frac{\dot{K}}{K}+\frac{w L}{p Q} \frac{\dot{L}}{L}
$$

y como $p=c$ en competencia, (6) se puede reducir a:

$$
\frac{\dot{Q}}{Q}=\frac{\dot{A}}{A}+\frac{r K}{c Q} \frac{\dot{K}}{K}+\frac{w L}{c Q} \frac{\dot{L}}{L}
$$

En (7), $\frac{w L}{c Q}$ es la participación del valor del trabajo en el valor de la producción, en tanto que $\frac{r K}{c Q}$ es la participación de la compensación al capital, y que en competencia perfecta se denota por $\tilde{S}_{l}$ y $\tilde{S}_{k}$, respectivamente. Sin embargo, como la suma de las 
participaciones del capital y el trabajo en la producción es igual a uno, $\tilde{S}_{l}+\tilde{S}_{k}=1$, y de esto último $\tilde{S}_{k}=1-\tilde{S}_{l}$. Así entonces, de (7) tenemos:

$$
\frac{\dot{Q}}{Q}=\frac{\dot{A}}{A}+\tilde{S_{K}} \frac{\dot{K}}{K}+\tilde{S_{l}} \frac{\dot{L}}{L}
$$

Esto es:

$$
\frac{\dot{Q}}{Q}=\frac{\dot{A}}{A}+\left(1-\tilde{S}_{l}\right) \frac{\dot{K}}{K}+\tilde{S}_{l} \frac{\dot{L}}{L}
$$

Si se resuelve la expresión entre paréntesis y se toma factor común $\tilde{S}_{l}$, tenemos:

$$
\left[\frac{\dot{Q}}{Q}-\frac{\dot{K}}{K}\right]=\frac{\dot{A}}{A}+\tilde{S}_{l}\left[\frac{\dot{L}}{L}-\frac{\dot{K}}{K}\right]
$$

Despejando $\frac{\dot{A}}{A}$ de (10) se obtiene el residuo de Solow bajo competencia perfecta:

$$
\left[\frac{\dot{Q}}{Q}-\frac{\dot{K}}{K}\right]-\tilde{S}_{l}\left[\frac{\dot{L}}{L}-\frac{\dot{K}}{K}\right]=\frac{\dot{A}}{A}
$$

El residuo mide el cambio del producto no explicado por el cambio en el trabajo o el capital. Pero un hecho relevante en la ecuación consiste en que el residuo de Solow es independiente de la razón producto/capital en industrias competitivas (Hall, 1988). 


\subsection{Generalización del Residuo de Solow}

En competencia imperfecta, bajo monopolio u oligopolio, el precio de mercado excede al costo marginal, es decir $p-c>0$. Si este exceso del precio sobre el costo marginal se divide por $p$, se obtiene el índice de Lerner de poder de mercado con un estricto valor positivo, $\frac{p-c}{p}=\beta$, indicador a estimar en el modelo más adelante.

En competencia perfecta, la participación relativa del trabajo en el producto es $\tilde{S}_{l}=\frac{w L}{c Q}$; al multiplicar y dividir por $p$, $\tilde{S}_{l}=\frac{w L}{c Q} \frac{p}{p}=\frac{p}{c} \frac{w L}{p Q}$, que es representado por $\tilde{S}_{l}=\frac{p}{c} S_{l}$. Esta última expresión se puede escribir como $\tilde{S}_{l}=\frac{S_{l}}{\frac{c}{p}}$. Si a $c$ se suma y resta $p$, entonces $\tilde{S}_{l}=\frac{S_{l}}{\frac{c-p+p}{p}}=\frac{S_{l}}{\frac{p}{p}-\frac{p-c}{p}}$, y así:

$\tilde{S}_{l}=\frac{S_{l}}{1-\beta}=(1-\beta)^{-1} S_{l}$

Reemplazando (12) en (11) se obtiene:

$\frac{\dot{A}}{A}=\left[\frac{\dot{Q}}{Q}-\frac{\dot{K}}{K}\right]+(1-\beta)^{-1} S_{l}\left[\frac{\dot{L}}{L}-\frac{\dot{K}}{K}\right]$

Si (13) es multiplicada por $(1-\beta)$ :

$\frac{\dot{A}}{A}(1-\beta)=\left[\frac{\dot{Q}}{Q}-\frac{\dot{K}}{K}\right]-\beta\left[\frac{\dot{Q}}{Q}-\frac{\dot{K}}{K}\right]-S_{l}\left[\frac{\dot{L}}{L}-\frac{\dot{K}}{K}\right]$ 
Ordenando términos:

$$
\left[\frac{\dot{Q}}{Q}-\frac{\dot{K}}{K}\right]-S_{l}\left[\frac{\dot{L}}{L}-\frac{\dot{K}}{K}\right]=\frac{\dot{A}}{A}(1-\beta)+\beta\left[\frac{\dot{Q}}{Q}-\frac{\dot{K}}{K}\right]
$$

El lado izquierdo de (15) es una generalización del original residuo de Solow desde la perspectiva del índice de Lerner, que bajo competencia imperfecta no es independiente de la tasa de crecimiento de la razón producto/capital; sin embargo, el progreso técnico es ahora procíclico, porque en competencia imperfecta el precio excede al costo marginal y el índice de Lerner es mayor que cero. Bajo competencia imperfecta se presenta correlación positiva entre el residuo de Solow y la tasa de crecimiento de la razón producto/capital; es precisamente esta correlación la que permite estimar el margen precio-costo marginal o índice de Lerner para la industria textil colombiana.

Bajo competencia perfecta, el índice de Lerner es cero y (15) es equivalente a (11). Si el índice de Lerner es positivo, existe poder de mercado en la industria y con él beneficios adicionales sobre el nivel de competencia. Este poder permite, en caso de monopolio, obtener beneficios adicionales o rentas monopolísticas (Stiglitz, 2005).

\section{MODELO EMPÍRICO, CONSTRUCCIÓN DE VARIABLES Y ESTIMACIÓN}

\subsection{Modelo Empírico}

El modelo empírico para la estimación del índice se basa en (15). El modelo econométrico es $r_{t}=c_{1}+c_{2} q_{t}+v_{t}$, donde $r_{t}=\left[\frac{\dot{Q}}{Q}-\frac{\dot{K}}{K}\right]-S_{l}\left[\frac{\dot{L}}{L}-\frac{\dot{K}}{K}\right], r_{t}$ es el residuo de Solow en el año t, la parte izquierda de (15). 
$c_{1}=\frac{\dot{A}}{A}(1-\beta) ; c_{1}$ es la medida del progreso técnico ajustado por el índice de Lerner, es constante en el modelo y representa el intercepto, $c_{2}=\beta$; el coeficiente de regresión $c_{2}$, es el índice de Lerner. Un valor positivo indica que la industria tiene poder de mercado. La teoría económica sugiere que el valor de $c_{2}$ está comprendido entre cero y uno, $q_{t}=\left[\frac{\dot{Q}}{Q}-\frac{\dot{K}}{K}\right] \cdot q_{t}$ es la tasa de crecimiento de la razón producto/capital en el año t.

En la estimación del modelo se supone que el termino error $v_{t}$ presenta distribución normal con media cero $E\left(v_{t}\right)=0$, y varianza $\sigma^{2}$ constante (homoscedasticidad). No existe autocorrelación de ninguna orden en los términos error. Además, la variable explicativa no está correlacionada con el término de error.

Sin embargo, debe observarse que cualquier choque causa un cambio en la tasa de crecimiento de la relación capital-producto y un cambio menos que proporcional en la tasa de crecimiento de la relación capital-trabajo. Esto genera un cambio simultáneo en la variable independiente del modelo, la cual es la tasa de crecimiento de la productividad total factorial. Por consiguiente, habría simultaneidad entre las variables, lo cual crea correlación entre el término de error y la variable independiente del modelo, llevando a que la estimación por mínimos cuadrados ordinarios de los parámetros del modelo pueda ser inconsistente, ineficientes y sesgada (Linnosmaa, et.al., 2004; Schmidt, 2005). La propiedad de la consistencia depende de que $E\left(v_{t} / q_{t}\right)=0$, si este supuesto no se cumple, $q_{t}$ es endógena. Para detectar la endogeneidad de la variable explicativa $q_{t}$ se aplica la prueba Hausman utilizando variable instrumental.

El instrumento $\Delta z_{t}$ debe ser exógeno, esto es, no estar correlacionado con $v_{t}$ (es decir $\left.\operatorname{corr}\left(\Delta z_{t} / v_{t}\right)=0\right)$, pero debe ser relevante, es decir fuertemente correlacionado con la variable endógena $\operatorname{corr}\left(\Delta z_{t} / q_{t}\right) \neq 0$. Se utiliza la renta nacional disponible como instrumento, porque afecta a la tasa de crecimiento de la razón pro- 
ducto/capital a través del efecto sobre la demanda interna textil (aunque no todo los productos demandados son de producción nacional), pero no tiene efectos, presumiblemente, sobre el progreso técnico de la industria.

\subsection{Construcción de Variables}

Los datos son agregados de las empresas de la industria textil que ofrecen información al DANE por medio de la Encuesta Anual Manufacturera. En la construcción de las variables del modelo, se calcula primero la tasa de crecimiento del producto (del valor agregado), del stock de capital y del trabajo. Posteriormente, para calcular la productividad, se tomó la diferencia entre la tasa de crecimiento del producto y del stock de capital, y entre la tasa de crecimiento del trabajo y el stock de capital; esta última diferencia es ponderada por la participación del trabajo en el valor de la producción (Harberger, 1969). La productividad se calcula utilizando el lado izquierdo de (15). En la Tabla 1 se presentan las series obtenidas para estimar el modelo empírico.

Tabla 1. Series para la estimación del modelo. Fuente: Autores

\begin{tabular}{cccccc}
\hline Año & rt & Qt & Año & rt & qt \\
\hline 1975 & $-0,172$ & $-0,205$ & 1991 & $-0,156$ & $-0,158$ \\
1976 & 0,242 & 0,245 & 1992 & $-0,127$ & $-0,034$ \\
1977 & 0,100 & 0,095 & 1993 & 0,006 & $-0,021$ \\
1978 & 0,040 & 0,000 & 1994 & 0,011 & 0,001 \\
1979 & $-0,036$ & $-0,054$ & 1995 & 0,063 & 0,033 \\
1980 & $-0,189$ & $-0,267$ & 1996 & 0,065 & 0,028 \\
1981 & $-0,152$ & $-0,194$ & 1997 & $-0,102$ & $-0,083$ \\
1982 & $-0,044$ & $-0,126$ & 1998 & $-0,025$ & $-0,078$ \\
1983 & $-0,009$ & $-0,074$ & 1999 & $-0,129$ & $-0,170$ \\
1984 & 0,177 & 0,178 & 2000 & 0,131 & 0,067 \\
1985 & 0,088 & 0,061 & 2001 & $-0,056$ & $-0,064$ \\
1986 & $-0,028$ & $-0,038$ & 2002 & $-0,053$ & $-0,057$ \\
1987 & 0,035 & 0,033 & 2003 & $-0,016$ & 0,000 \\
1988 & 0,208 & 0,190 & 2004 & 0,059 & 0,056 \\
1989 & 0,007 & $-0,001$ & 2005 & $-0,002$ & 0,012 \\
1990 & $-0,011$ & $-0,033$ & 2006 & 0,037 & 0,030 \\
\hline
\end{tabular}




\subsection{Estimación}

El primer trabajo a realizar con series de tiempo es determinar si son estacionarias y si entre ellas existe una relación de cointegración. Si se realiza la regresión de una serie de tiempo no estacionaria sobre otra no estacionaria se puede obtener una regresión espuria, en apariencia, una relación estadísticamente significativa cuando en realidad no sucede así.

La estacionariedad se refiere a que el proceso estocástico generador de la serie tiene media y varianza constante en el tiempo (no dependen del tiempo) y el valor de la covarianza entre dos períodos depende del rezago y no del tiempo. Si dos series no estacionarias presentan entre ellas una relación de equilibrio o de largo plazo, se dice que son cointegradas. Para verificar la cointegración se hace una prueba de raíz unitaria a los residuos. Si se verifica que dos o más variables integradas del mismo orden están cointegradas, se asegura una relación entre ellas no espuria, y es además estacionaria, es decir, presenta equilibrio estable y no acumula perturbaciones de períodos anteriores.

A continuación se realiza el análisis de la estacionariedad de las series y la relación de cointegración. Para el efecto, se aplica la prueba de raíces unitarias desarrollada por Phillips - Perron sobre las series $r_{t}$ y $q_{t}$. En la Tabla 2 se presentan los resultados. Para sendas variables, el p-valor del contraste es menor que 0,05 e indica que las series son estacionarias. Se infiere que las series son integradas de orden cero. Adicionalmente, se debe determinar si entre $r_{t}$ y $q_{t}$.existe una relación de cointegración. La prueba consiste en determinar la estacionariedad de los residuos. Para ello, se realiza la regresión de $r_{t}$ contra $q_{t}$ con el fin de generar los residuos de la regresión y verificar la presencia de raíz unitaria. Como el resultado en p-valor es menor que 0.05 , se concluye que los residuos son estacionarios. Por lo tanto, al ser las series $r_{t}$ y $q_{t}$ integradas del mismo orden y los residuales estacionarios, las variables del modelo cointegran y la estimación del modelo $r_{t}=c_{1}+c_{2} q_{t}+v_{t}$ muestra una relación no espuria. 
Antes de estimar el modelo, es necesario verificar la no presencia de correlación entre la variable explicativa y el término error, es decir la endogeneidad de $q_{t}$. Para ello se realiza la prueba de endogeneidad de Hausman utilizando como variable instrumental a la renta nacional bruta disponible $(Y d t)$. De acuerdo con la Tabla 2, donde se presenta el test de raíces unitarias Phillips-Perron, la serie Ydt es integrada de orden cero.

Tabla 2. Análisis de series, endogeneidad y quiebre estructural. Fuente: Autores

\begin{tabular}{|c|c|c|c|}
\hline Variable/ Modelo & Prueba & Resultado & Observación \\
\hline $\begin{array}{c}\mathrm{rt} \\
\mathrm{qt} \\
\text { residuales }\end{array}$ & $\begin{array}{c}\text { Test raíz } \\
\text { unitaria } \\
\text { Phillips-Perron }\end{array}$ & $\begin{array}{l}\text { P-valor } 0,0005 \\
\text { P-valor } 0,0008 \\
\text { P-valor } 0,0006\end{array}$ & $\begin{array}{c}\text { Serie estacionaria } \\
\text { Serie estacionaria } \\
\text { Serie estacionaria } \\
\text { Las variables } \\
\text { cointegran }\end{array}$ \\
\hline $\begin{array}{c}\text { Variable } \\
\text { instrumental } Y d t\end{array}$ & $\begin{array}{c}\text { Test raíz } \\
\text { unitaria } \\
\text { Phillips-Perron }\end{array}$ & $\mathrm{P}$-valor 0,0011 & Serie estacionaria \\
\hline $\begin{array}{c}\text { Prueba de } \\
\text { endogeneidad: } \\
q_{t}=a_{1}+a_{2} \mathrm{Yd}_{t}+v_{t}\end{array}$ & $\begin{array}{l}\text { Prueba } \\
\text { Hausman }\end{array}$ & $\begin{array}{l}\text { Coeficiente } Y d t \\
\text { P-valor } 0,1640\end{array}$ & $\begin{array}{c}\text { Coeficiente no } \\
\text { significativo al } 95 \%\end{array}$ \\
\hline Residuales & $\begin{array}{l}\text { Test raíz } \\
\text { unitaria }\end{array}$ & P-valor 0,0022 & $\begin{array}{l}\text { Residuales } \\
\text { estacionarios }\end{array}$ \\
\hline$r_{t}=c_{1}+a_{2} q_{t}+$ resid & M.C.O. & $\begin{array}{c}\text { P-valor } 0,1383 \\
R^{2}=0,92 \\
\text { DW }=1,956\end{array}$ & $\begin{array}{c}\text { Residuales no } \\
\text { significativos al } \\
95 \% . \\
\text { La variable qt no es } \\
\text { endógena }\end{array}$ \\
\hline $\begin{array}{c}\text { Ruptura estructural } \\
\text { para } 1992\end{array}$ & Prueba Chow & P-valor $=0,000$ & $\begin{array}{c}\text { Existe cambio } \\
\text { estructural en } 1992\end{array}$ \\
\hline
\end{tabular}

Con la estimación por mínimos cuadrados ordinarios de la regresión entre la variable endógena $q_{t}$ respecto a su instrumento $Y d t$ y los residuales se determina la estacionariedad y endogeneidad, según el contraste Hausman. En la Tabla 2 se presentan los resultados. Se observa que el coeficiente de la variable $Y d t$ no es estadísticamente, significativo. Teniendo en 
cuenta los resultados anteriores, se estima el modelo original incluyendo los residuales como variable explicativa. Si los residuales son significativos se concluye que la variable explicativa $q_{t}$ es endógena. Se estima la regresión de $r_{t}$ con respecto a $q_{t} \mathrm{y}$ residuales, incluyéndose intercepto.

El resultado se presenta en la Tabla 3. Se observa como el coeficiente de la variable "resid" no es significativo al 95\%, y aunque los demás estadísticos, $R^{2}$ y el Durbin-Watson, son apropiados, se infiere desde el contraste de Hausman que el regresor $q_{t}$ no es endógeno. La prueba Chow detecta ruptura estructural en el año 1992, por lo que se incluye en la estimación del modelo una dummy aditiva para este año que toma el valor de 1 , y cero en el resto del período caso

Tabla 3. Estimación del modelo y diagnóstico. Fuente: Autores

\begin{tabular}{|c|c|c|c|}
\hline Estimación & Método & Resultado & Observación \\
\hline$r_{t}=c_{1}+c_{2} q_{t}+c_{3} D+v_{t}$ & M.C.O & $\begin{array}{c}c_{1}=0,020143 \\
\text { p-valor }=0,0001 \\
c_{2}=0,899 \\
\text { p-valor }=0,000 \\
c_{3}=-0,0117 \\
\text { P-valor }=0,0001\end{array}$ & $\begin{array}{c}\text { Coeficientes } \\
\text { significativos, } \\
\text { Indice de Lerner } \\
\text { positivo, } 0<\beta<1\end{array}$ \\
\hline $\begin{array}{l}\text { Normalidad de los } \\
\text { residuos }\end{array}$ & $\begin{array}{c}\text { Test de } \\
\text { normalidad }\end{array}$ & $\begin{array}{c}\text { JB: } 0,448183 \\
\text { Prob. } 0,799\end{array}$ & $\begin{array}{c}\text { Se acepta } \\
\text { formalmente la } \\
\text { normalidad de los } \\
\text { residuos } \\
\text { P-valor }>0.05\end{array}$ \\
\hline Autocorrelación & $\begin{array}{l}\text { Breusch- } \\
\text { Godfrey }\end{array}$ & $\begin{array}{c}\text { F:1,17108 } \\
\text { P-valor: 0,325 } \\
\text { Chi cuadrado: } \\
2,55 \\
\text { P-valor: } 0,2779\end{array}$ & $\begin{array}{l}\text { P-valor }>0.05 . \\
\text { No estructura auto- } \\
\text { regresiva. }\end{array}$ \\
\hline Heteroscedasticidad & Test White & $\begin{array}{c}\text { F: } 0,7426 \\
\text { P-valor: } 0,5338 \\
\text { Productos } \\
\text { cruzados: } 2,36 \\
\text { P-valor: } 0,5051\end{array}$ & $\begin{array}{c}\mathrm{Al} 95 \% \text { los p- } \\
\text { valores de la F y } \\
\text { términos cruzados } \\
\text { son mayores al } \\
5.00 \% \\
\text { Homoscedasticidad }\end{array}$ \\
\hline
\end{tabular}




\section{ANÁLISIS DE RESULTADOS Y DISCUSIÓN}

El presente estudio permite verificar el comportamiento asumido por la industria textil en Colombia entre 1975 y 2006. En la evolución de la serie $r_{t}$, se observa cómo el residuo de Solow en 1975 fue $-0,17$ y durante varios periodos presentó valores negativos, para cerrar en 0,03 en 2006. Es de anotar que durante las décadas de 1970 y 1980, dicha variable mostró un comportamiento altamente heterogéneo, posiblemente asociado a la estructura propia del sector, determinada por políticas proteccionistas. Por el contrario, la década de 1990 marcó la estabilización del progreso técnico, cuyas fluctuaciones fueron menos notorias, a pesar de que evidenció un leve decrecimiento principalmente durante la desaceleración y recesión económica a finales de la década de 1990.

La caída en el crecimiento del progreso técnico concuerda con las crisis más agudas que se presentaron durante el período analizado; la crisis de acumulación de capital de finales de la década de 1970 y principios de la década de 1980, y la recesión de 1999 (Departamento Nacional de Planeación, 2003). La oscilación de la serie indica que el progreso técnico incorporado en el sector asume una senda altamente fluctuante hasta la década de 1990, pero se estabiliza luego de la apertura de 1990, tal y como se evidencia en la Fig. 1.

Del mismo modo, la tasa de crecimiento de la relación producto capital pasó de valores negativos, -0,20 en 1975 a 0,03016 en 2006. Esto indica que los procesos productivos han sido altamente intensivos en el uso del factor trabajo, hecho que se evidencia a partir de los valores negativos alcanzados por el crecimiento de dicha variable durante varios años del periodo analizado. No obstante, a partir del año 2003 manifestó un leve crecimiento, lo cual determinó que para el año 2006 el valor alcanzado por la razón anteriormente mencionada fuera positivo. Sin embargo, este leve crecimiento denota la insuficiencia de los esfuerzos realizados por los empresarios en aras de incrementar la intensidad en el uso del capital, pues la evolución de la razón producto-capital aun manifiesta valores muy bajos, tal y como se visualiza en la Fig. 2. 


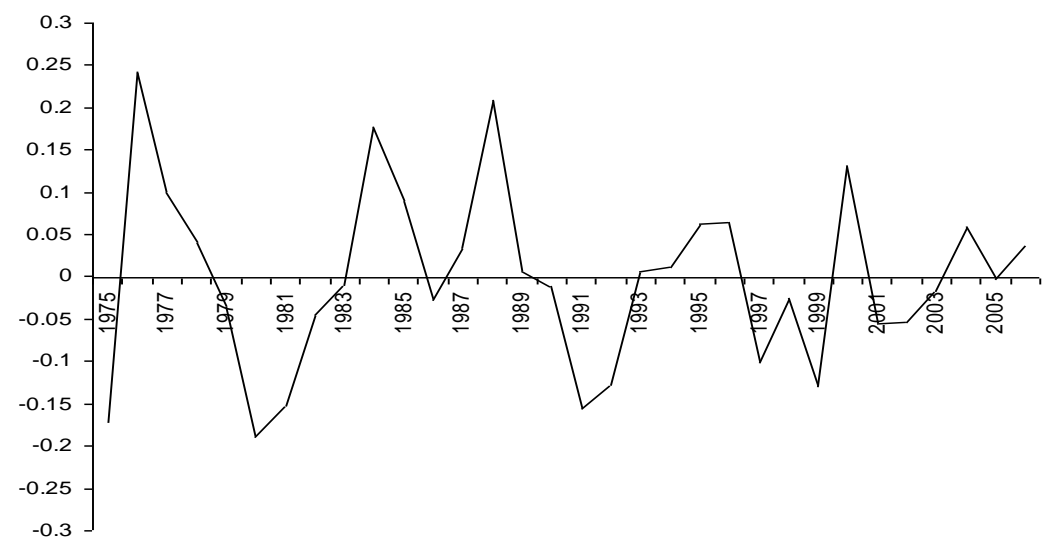

Fig. 1. Crecimiento del residuo de Solow (progreso técnico) del sector textil colombiano, 1975-2006. Fuente: Autores

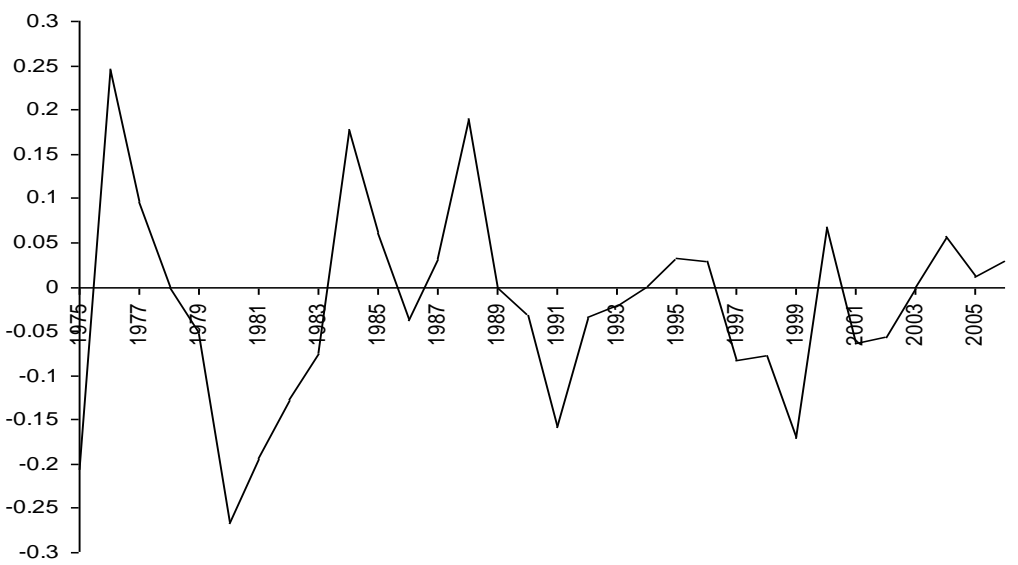

Fig. 2. Crecimiento de la relación capital/trabajo del sector textil colombiano, 1975-2006. Fuente: Autores

Entre los resultados más relevantes cabe destacar el comportamiento del poder de mercado desde el punto de vista de la producción. El índice de Lerner para la industria textil colombiana entre 1975 y 2006 es positivo y significativamente diferente de cero. El valor estimado es de 0,899 permitiendo afirmar la 
existencia de poder de mercado en la industria textil. En el período de 1975 a 1990, la industria textil estaba altamente concentrada, pues era un sector cerrado al comercio internacional y protegido por el Estado con barreras al comercio. Se contaba con la participación de pocas empresas que jalonaron la industrialización en el país. La protección generaba procesos intensivos en mano de obra, consecuentes con una mayor generación de empleo en el país y los pocos incentivos para aumentar la eficiencia productiva. Luego de 1990, se da la apertura económica y la entrada de importaciones al mercado nacional; sin embargo, la competencia en el país no aumentó como para afirmar que los procesos tendieron a mercados competitivos. Al contrario, la industria se transformó y con las mejoras en los procesos de producción dejó por fuera varias empresas, que por problemas internos, como el alto pasivo pensional, procesos altamente intensivos en mano de obra y obsolescencia tecnológica, salieron del mercado o fueron absorbidas por las que sobrevivieron al proceso de apertura.

Las razones anteriores explican la alta concentración de la producción en este sector, aunque esto no se refleja en altas tasas de ganancia, debido en parte a la competencia por parte de las importaciones de textiles, tanto legales como ilegales. Asimismo surgieron nuevas empresas que aprovecharon el cambio técnico y de fisionomía del producto, al pasar de procesos de elaboración de tejido plano a de tejido de punto, para posicionarse tanto en el mercado nacional como internacional. En cuanto al comportamiento de la demanda de productos textiles producidos en Colombia, la elasticidad precio de la demanda (calculada como el inverso del índice de Lerner) arroja un resultado de 1,112; siendo elásticos ante el precio los productos de esta industria, es decir, ante una variación de $1 \%$ en el precio, la cantidad demandada varía en un $1,112 \%$ en el sentido inverso del cambio en el precio. Estos resultados se presentan en la Tabla 4.

El valor estimado del índice de Lerner implica que el costo marginal de la industria ascienda a 0,101 mientras que el markup de Hall, definido como la relación precio/costo marginal, es de 9,9. Se observa entonces, que la industria textil está en el tramo elástico de la curva de la demanda, comprobando el comportamiento como bien ordinario, es decir, la cantidad 
demandada aumenta más que proporcionalmente en la medida en que disminuye el precio.

Tabla 4. Poder de mercado e indicadores estimados. Fuente: Autores

\begin{tabular}{ccc}
\hline Variable & Definición & Resultado \\
\hline Índice de Lerner & $\beta=\frac{p-c}{p}$ & 0,899 \\
Costo marginal & $c$ & 0,101 \\
Markup de Hall & $\mu=\frac{P}{c}$ & 9,9 \\
$\begin{array}{c}\text { Elasticidad precio de la } \\
\text { demanda }\end{array}$ & $|\varepsilon|=\frac{1}{\beta}$ & 1,112 \\
$\begin{array}{c}\text { Productividad total de los } \\
\text { factores promedio anual }\end{array}$ & $\frac{\dot{A}}{A}$ & $19,86 \%$ \\
\hline
\end{tabular}

En términos generales, estos resultados manifiestan un alto grado de poder de mercado, concentración de la producción en unas pocas empresas y restricción de la competencia representada en barreras a la entrada, monopolios naturales o distorsiones como monopolios legales y algunas barreras arancelarias que siguen protegiendo a la industria nacional. Así entonces, es posible aseverar que en la industria textil existe un alto poder de mercado, a pesar del cual no se puede asegurar que los márgenes de ganancias sean igualmente altos dado que no es posible observar el comportamiento de la curva de costo medio con relación a la curva de demanda. Asimismo se puede concluir que el problema del sector está marcado por una precaria inversión en la mejora de los procesos de producción con vistas a hacerlo más competitivo en los mercados internacionales.

La tasa de crecimiento anual promedio de la productividad técnica factorial es de $19,86 \%$. Al comparar este indicador con el poder de mercado del sector y la razón de crecimiento de la razón capital trabajo, se puede afirmar que la productividad total es procíclica y permite calcular el índice de Lerner como medida del poder de mercado, sin contradecir las implicaciones del modelo de Solow. 


\section{CONCLUSIONES}

Este artículo presenta el desarrollo teórico de un modelo para estimar el margen precio-costo marginal o índice de Lerner para la industria textil colombiana. Los cálculos arrojan un índice de Lerner de 0,899 , del que se deduce el costo marginal $(0,101)$ y el markup de Hall $(9,9)$. En conclusión, se verifica la existencia de poder de mercado en la industria textil colombiana, al estimarse un índice de Lerner mayor que cero y un markup de Hall mayor que uno.

El poder de mercado en la producción de textiles en Colombia, implica transferencia de excedente del consumidor al productor, menor demanda de factores de producción respecto de la situación de competencia perfecta, por lo que habría pérdida irrecuperable de eficiencia. La productividad total de los factores en la industria textil colombiana, con un promedio anual de crecimiento de $19,86 \%$, es altamente procíclica. La tasa de crecimiento de la razón producto/capital explica significativamente las variaciones en el residuo de Solow.

Con el modelo desarrollado se determinó el nivel de concentración en la industria textil, sin embargo, no fue posible obtener el precio final y la tasa de ganancia del sector, debido a la falta de información para estimar el costo medio de la industria.

\section{REFERENCIAS}

Gallego, J., (2009); Modelo teórico para la determinación del poder de mercado, Tecno Lógicas, 22: 189-208.

Hall, R., (1988); The relation between price and marginal cost in U.S. industry. The journal of Political Economy, 96(5): 921-947.

Harberger, A., (1969); La tasa de crecimiento del capital en Colombia. Revista de Planeación y Desarrollo. Departamento Nacional de Planeación, 1(3): 13-42.

Linnosmaa, I., Hermans, R., Hallinen, T., (2004); Price-cost marginal in the pharmaceutical industry. Empirical evidence from Finland. European Journal Health Economics, 5: 122-128. 
Romer, D., (2001); Macroeconomía Avanzada. España: Mc Graw -Hill.

Schmidt, S. J., (2005); Econometría. México: Mc Graw -Hill.

Solow, R., (1957); Technical change and the aggregate production function, Review of economics and statistics, 39(3): 312-320.

Stiglitz, J. E., (1998); Microecnomía. España: Ariel. 
